\title{
CONSTRUCCIÓN DE UN CORPUS LITERARIO LATINOAMERICANO Y CARIBEÑO (CLLYC) EN IMPRESO PARA SUS BÚSQUEDAS EN SOPORTE DIGITAL
}

Adrián R. Vila

editorvila@gmail.com

\section{INTRODUCCIÓN}

El presente paper se propone como metodología de construcción de un corpus literario para la realización de búsquedas de títulos y autores en el marco de la transposición de impreso a digital. Una de las investigaciones que nos propusimos realizar es la del estado de la cuestión de la transposición a digital de la literatura latinoamericana y caribeña editada en impreso. Ahora bien: una vez establecida la dilucidación terminológica y conceptual que delimite definiciones y términos teóricos trabajados: Canon / Corpus; Hispanoamérica / Iberoamérica / América Latina / Caribe; Literatura latinoamericana y caribeña (entre otras), es estructural a los objetivos de la investigación el diseño de un corpus literario latinoamericano y caribeño (CCLyL) para las búsquedas. Una vez definido y delimitado dicho corpus, establecer en qué plataformas y bibliotecas se deberían efectuar las búsquedas. Finalmente, cómo efectuarlas prácticamente y algunos resultados obtenidos en función de la dilucidación de estrategias de la industria editorial en digital para la transposición a digital de literatura impresa. Se detallan etapas y pasos cumplimentados a los efectos de la mencionada construcción. 


\section{DELIMITACIÓN DE TÉRMINOS Y DISEÑO DE UN CORPUS LITERARIO LATINOAMERICANO Y CARIBEÑO (CLLYC). ¿CORPUS DE QUÉ? ¿CORPUS PARA QUÉ?}

En principio, el adjetivo "latina" proviene del legado imperial francés como forma de incluir a Francia entre los países con influencia en América, excluyendo a las potencias anglosajonas. En 1836, el economista político Michel Chevalier publicaba en París las crónicas de sus viajes por América, continente en el que se replicaban a su entender las diferencias étnicas que se daban en Europa de la época:

Las dos ramas, latina y germana, se reproducen en el Nuevo Mundo. América del Sur es -como la Europa meridional-, católica y latina. La América del Norte pertenece a una población protestante y anglosajona (apud ARDAO, 1996, p. 55).

Esta primera idea, "creadora" del concepto, inicia la evolución del mismo por oposición a otros conceptos: latina por oposición a sajona. España impulsó el término "Hispanoamérica" como marca de sus derechos por antigüedad, tradición, influencia, concepción geopolítica y continuidad de su presencia en la región desde 1492. A su vez, "Iberoamérica" fue la forma que encontraron España y Portugal en función de empalmar en una identidad común a las antiguas metrópolis de la península ibérica con sus viejas colonias (incorporando a Brasil) y, a su vez, excluir de la identificación, sobre todo, a Francia.

Una primera aproximación de carácter geográfico a la categoría "América Latina" designa las partes del nuevo mundo que fueron colonizadas por naciones de la Europa latina como España, Francia y Portugal. Sin embargo, un agrupamiento basado en regiones geográficas trae aparejadas inconsistencias de demarcación. Existen zonas del Caribe, Centro y Sudamérica que fueron dominadas por Inglaterra u Holanda (potencias coloniales no latinas): Antigua y Barbuda, Bahamas, Barbados, Belice, Dominica, Granada, Guayana, Jamaica, Saint Kitts y Nevis, San Vicente y las Granadinas, Santa Lucía, Surinam, Trinidad y Tobago. Del mismo modo, hay zonas de Norteamérica en Canadá y Estados Unidos que fueron colonizadas por Francia y España, pero no se consideran latinoamericanas: Quebec, Luisiana, Florida, las regiones del Sur norteamericano anexionadas por EE.UU. y de antiguo dominio mexicano. Pero, asimismo, para analistas como Rouquié (1989, p. 17), se trataría de un fallido "concepto cultural", que contendría a las naciones de cultura 
latina de América. Pero este tipo de encuadres traerían aparejadas otras inconsistencias: la inclusión de Canadá y su provincia francohablante de Quebec, "infinitamente más latina que Belice y tanto como Puerto Rico, estado libre asociado de Estados Unidos", pero a los que nunca nadie ha pensado incluirlos, "ni siquiera al nivel de su provincia francohablante, en su subconjunto latinoamericano” (ROUQUIÉ, 1989, p. 17).

A su vez, destacamos que el análisis previo nos permite señalar las imprecisiones y la ambigüedad de la etiqueta "América Latina", al dilucidar que este concepto no es "ni plenamente cultural ni solamente geográfico" (ROUQUIÉ, 1989, p. 17). La noción de existencia de América Latina definida por oposición y desde afuera es la que prima en este trabajo, es decir, la mayoría de las veces utilizamos esta categoría como forma de oponer a los países de la región con otros países. Y, a la vez, como forma de identificación de producciones culturales de los artistas de la región en relación con las producciones de otras regiones. Aunque en el área cultural, "pese a la multiplicación de organismos integradores desde los años cincuenta (OEA, CEPAL, ALALC, etc.), ni siquiera hemos logrado establecer entre los países de América Latina formas de colaboración duradera y de conocimiento recíproco" (GARCÍA CANCLINI, 1995, p. 149). En este plano, la circulación intrarregional de bienes culturales sigue siendo deficiente, aunque el crecimiento de algunas producciones en el ecosistema digital indica una tendencia que puede facilitar la integración en algunas disciplinas. Utilizaremos la categoría América Latina porque se ofrece como un término "cómodo", sin ignorar "sus límites y sus ambigüedades” (ROUQUIÉ, 1989, p. 20) y demarcando por oposición:

\footnotetext{
El término no alcanza a países ubicados en el continente como Belice, Surinam, Guyana, Guyana Francesa, Estados Unidos o Canadá. Tampoco a las islas caribeñas anglosajonas como Jamaica, Barbados, Antigua y Barbuda, Santa Lucía, San Kitts y Nevis, San Vicente y las Granadinas, Dominica (VARDA, 2015).
}

Aunque, ya desde 1948 las Naciones Unidas con su proceso de regionalización fue dando lugar a la creación de organismos como CEPAL (Comisión Económica para América Latina y el Caribe), ILPES (Instituto Latinoamericano y del Caribe de Planificación Económica y Social), CELADE (Centro Latinoamericano de Desarrollo Empresarial), CLACSO (Consejo Latinoamericano de Ciencias Sociales) que fueron incorporando la idea de bloque regional compuesto por América Latina y el Caribe, a su vez, se fueron dando incorporaciones sucesivas a partir 
de la creación de organismos aglutinadores posteriores: la Comunidad de Estados Latinoamericanos y Caribeños (CELAC) creada en 2011 (además de la Unión de Naciones Suramericanas -UNASUR- que está formada por 12 países soberanos independientes, en funciones desde 2011). ${ }^{1}$

Si las organizaciones globales (ONU, UNESCO, Banco Mundial) imponen lacategoría "América Latinayel Caribe" con finesdesimplificación estadística, la composición de la Comunidad de Estados Latinoamericanos y Caribeños (CELAC) demuestra que estos países la aceptan. Es decir, que el "autorreconocimiento de la etiqueta por los países implicados vía participación en la CELAC" (MALAMUD, 2015) funciona como uno de los criterios de demarcación. A esta vinculación entre América Latina y el Caribe en términos de políticas gubernamentales y diplomáticas le es subsidiaria la incorporación de la literatura francohablante del Caribe desde mediados de los años 1960 al concepto de "literatura latinoamericana", que sirvió como avanzada para una vinculación entre la literatura latinoamericana y el resto de la literatura caribeña. Vinculación articulada, sobre todo por dos tipos de dispositivos teóricos: a) por un lado, la historia común del Caribe insular anclada en rasgos económicosociales similares como lo son "economía de plantación, trata de esclavos, historia de cimarronaje, cultura de resistencia, incomunicación entre las islas y luchas entre las metrópolis" entre otros rasgos (PIZARRO, 1987, p. 25) y b) por otro lado, las relaciones históricas comunes del Caribe con zonas de la región latinoamericana, las formas de desarrollo cultural similares y la literatura, por la evidencia de temas y problemas, así como "articulaciones que son asumidas tanto en el caso de las literaturas del Caribe inglés y holandés como en el caso del francés y el español, tanto el papiamento o su equivalente de Surinam, en los términos en que se asumen en la literatura latinoamericana" (PIZARRO, 1987, p. 25). A su vez, estos rasgos de similitud en la organización de los sistemas literarios de América Latina y Caribe son expuestos por aquellos trabajos de este tipo de dispositivos teóricos del tipo b) que marcábamos previamente: existen en un primer nivel sistemas literarios ligados a la metrópolis (Gran Bretaña, Holanda, Francia); en un segundo nivel se dan otros sistemas

${ }^{1}$ Los países miembros de la CELAC son 33: Antigua y Barbuda, Argentina, Bahamas, Barbados, Belice, Bolivia, Brasil, Colombia, Costa Rica, Cuba, Chile, Dominica, Ecuador, El Salvador, Granada, Guatemala, Guyana, Haití, Honduras, Jamaica, México, Nicaragua, Panamá, Paraguay, Perú, República Dominicana, San Cristóbal y Nieves, San Vicente y las Granadinas, Santa Lucía, Surinam, Trinidad y Tobago, Uruguay y Venezuela. 
literarios en créole, papiamento, papiamen; e inclusive, en un tercer nivel, sistemas multilingües. Es decir, que la estructura de los sistemas literarios del Caribe, Antillas y Guayanas se muestra organizada con la misma disociación de las estructuras lingüísticas heredadas de la metrópolis que en América Latina.

Así, Cornejo Polar (1987, p. 131) destaca que el sistema literario latinoamericano se desarrolla en la tensión entre fuerzas que conducen a la integración y fuerzas que "tienden a desmembrarle". Pero, también, en el marco de las contradicciones propias al interior del sistema literario combinadas con "las fluctuaciones que dependen de la hegemonía que pueda adquirir en determinado momento una de esas dos fuerzas". Las operaciones de integración literaria (y cultural) latinoamericana se iniciaron con un nivel inicial de integración de las letras en castellano, luego pasaron a un segundo nivel de integración de las letras americanas en castellano y portugués (que incorporó la literatura brasileña), para derivar en un tercer nivel de integración de las literaturas de todas las lenguas latinas con la inclusión de las literaturas en francés (especialmente, de Haití). Ardao (1996, p. 33-34) propone un cuarto nivel de integración, "supernumerario" con la incorporación de "las letras americanas de otras lenguas correspondientes a su área: autóctonas hacia un extremo, de procedencia europea no latina hacia otro" en el contexto de un proceso no completado "a cabalidad”. En este cuarto nivel de integración, tendríamos un sistema literario latinoamericano integrado por todas las literaturas en las distintas variedades del castellano y del portugués de América Latina, francés, inglés, holandés, papiamento, guaraní, náhuatl, aymará, quiché, patois, créole, jorapa, portuñol y las heteroglosias de todas las regiones de América Latina, Caribe, Antillas y Guayanas. En este cuarto nivel, ya disponemos de un campo literario común latinoamericano y caribeño.

Lotman (2000) propone una descripción del funcionamiento de los sistemas culturales como poseedores de una dualidad que consiste en la articulación de dos tendencias dinámicas inversas: a) una que constituye a la cultura como repetición en otro plano organizativo de la persona aislada, pero, al mismo tiempo, b) como antítesis de la conciencia individual (mecanismo que debe suprimir las dificultades de la persona aislada). Es decir, que en ese doble movimiento los sistemas culturales pujan por sus dos tendencias intrínsecas: a la diversidad por un lado, y por el otro a la uniformidad. Promoción de más subsistemas y elementos componentes en función de la adquisición de equilibrios al interior del sistema, 
por un lado. En sentido inverso, una tendencia a la uniformización en función de la identidad más lograda de cada sistema, una tendencia a la autodescripción canónica con la elección de sus textualidades "mejores". Son las "metadescripciones" (LOTMAN, 2000, p. 128, 143, 145).

Entonces, tomando como préstamo esta perspectiva para la construcción de nuestro CLLyC, éste puede ofrecerse como un sistema compuesto de múltiples subsistemas diversos, autónomos e intercambiables. Esto también significa que para el CLLyC y por analogía con el sistema cultural lotmaniano, ningún componente es mayor/mejor/más importante que otros. Ahora bien, al mismo tiempo, el propio sistema genera formas de autodescripción, a las que Lotman llama "metadescripciones", que funcionan como modelos que tienen la necesidad dedescribirsea sí mismos. Estelenguaje metadescriptor produce elecciones de tipos de textos "mejores" para la descripción necesaria: en ese plano se desarrollaría la función canónica. El canon opera como forma jerarquizadora de textos, dejando afuera innumerables formas textuales "no correctas". Esta dinámica apunta a la uniformización cultural, al achicamiento del sistema. Y, en nuestro CLLyC, esas versiones textuales también conforman sus subsistemas a los que llamamos "segmentos del corpus" (ver Figura 1. Composición del CLLyC). Si el propio desarrollo de la cultura está asentado sobre la puja entre las formas sistémicas y las extrasistémicas, en el corpus desarrollado como CLLyC estas tensiones se expresan sin exclusiones. O sea: a grandes rasgos, los textos canónicos y no canónicos son sus subsistemas componentes.

Así, el CLLyC construido surge del relevamiento de fuentes documentales de este campo literario latinoamericano y caribeño (repertorios, ensayos, antologías, artículos académicos, diccionarios), literarias latinoamericanas heterogéneas, disímiles, distantes entre sí tanto en el tiempo como en las perspectivas y criterios de selección de autores y obras por las propias fuentes. Las listas de autores y obras fueron extraídas de fuentes que las aportan desde enfoques diversos y funcionan metodológicamente para las búsquedas como listados complementarios entre sí que ocupan las áreas de vacancia expuestas por las demás fuentes. Y asimismo, que funcionan metodológicamente como opuestos entre sí, lo que permite expresar al interior del CLLyC tanto la complejidad de las formas literarias de la región como algunas de las dispositividades materiales (CHARTIER, 1992) mediante las cuales circulan esas literaturas. 
- Se construyó la lista de 18 autores y 36 obras que Harold Bloom introduce en el segmento latinoamericano de su canon y los 5 autores y 7 obras que introduce en el segmento caribeño (West Indian) como parte de lo que llama "profecía canónica" de la "edad caótica" (siglo XX). Es decir: 43 títulos de 23 autores de El canon occidental de Harold Bloom (1995).

- El Diccionario de autores latinoamericanos realizado por César Aira aporta más de 1.300 autores y obras con un criterio de amplitud en la incorporación de autores: dramaturgos, poetas, novelistas, cuentistas, científicos, historiadores, cronistas, periodistas y ensayistas. Asimismo, nos provee de un segmento significativo de literatura brasileña (incorporación que no se realiza con frecuencia en los repertorios literarios latinoamericanos), pero no incorpora autores y obras precolombinos ni en otras lenguas que no sean castellano y portugués, así como no incluye autores surgidos a partir de 1985. Se transcribieron todos los autores y obras relevadas por Aira. Aporta 11.102 títulos de 1.322 autores.

- El Diccionario de la literatura latinoamericana de Susana Cella contribuye con un listado de autores y obras que funciona de manera complementaria con el Diccionario de autores latinoamericanos de César Aira: incorpora textos precolombinos y en lenguas no hispánicas y a los escritores nacidos hasta 1940 o, en caso de que hayan nacido después del 1940, que ya estuviesen fallecidos en 1998. La idea de "representatividad" de autores y obras prima sobre la "exhaustividad" (CELLA, 1998, p. VII). Aporta 4.737 títulos de 595 autores.

- La Encyclopedia of Latin American and Caribbean Literature 1900-2003, editada por Daniel Balderston y Mike Gonzalez, incorpora obras escritas entre esos años: 1900 y 2003, con lo cual también funciona como recorte temporal, además de geopolítico cultural.

- Saraus. Movimiento - Literatura - Periferia - São Paulo. Antología, de Lucía Tennina (compiladora) aporta autores y obras de poetas paulistas contemporáneos de la llamada "marginalia" y "estética marginal". Aporta 58 títulos de 38 autores.

- De la Tricolor a la Wiphala. Narrativa contemporánea de Bolivia, de Sergio Di Nucci, Nicolás Recoaro y Alfredo Grieco y Bavio (selección, edición y notas) contribuye con cuentos de 14 autores bolivianos nacidos entre 1956 y mediados de los años 1980, con la que articula una selección que expresa la emergencia de una nueva narrativa boliviana independiente, localizada tanto en la Ceja de El Alto pasando por la tropical selvática Santa Cruz de la Sierra y la geografía urbana de La Paz hasta el barrio de Liniers 
(Buenos Aires, Argentina), donde habita una de las concentraciones más grandes de bolivianos en el exterior. Aporta 67 títulos de 14 autores.

- McOndo (una antología de la nueva literatura hispanoamericana) editada por Alberto Fuguet y Alberto Gómez aporta a este CLLyC cuentos de aquellos autores incorporados a la antología por decisión programática nacidos desde 1959 "(que coincide con la siempre recurrida revolución cubana) a 1962 (que en Chile y en otros países, es el año en que llega la televisión)" (FUGUET, 1996, p. 9), además de autores "poco conocidos" hasta mediados de los años 1990 y cuya selección descarta, también por decisión programática, todo rastro de realismo mágico como poética. $A$ posteriori, Fuguet (TSANG, 2013) amplía las características poéticas de la generación literaria de esa antología a "lo híbrido, la mezcla". Pasada una década, Latinoamérica y su arte "han ido para allá": para McOndo. Y, en esa misma operación, incorpora al "gran autor McOndo": Roberto Bolaño, por "ultrarrealista” o "posmoderno". Ese listado de autores y de obras resultante de la antología $M c O n d o$ es el aporte a la construcción de este CLLyC. Aporta 17 títulos de 15 autores.

- El Manifiesto del Crack aporta una lista de seis autores mexicanos (Jorge Volpi, Pedro Ángel Palou, Eloy Urroz, Ignacio Padilla, Ricardo Chávez Castañeda y Vicente Herrasti), con un programa poético de una narrativa dislocada o desubicada del espacio y del tiempo de México, cuyas obras, todas posteriores a 1996, se caracterizan por una postura narrativa rupturista tanto del realismo mágico latinoamericano como de una cierta poética light representada por Ángeles Mastretta, Marcela Serrano, Laura Esquivel, Guadalupe Loaeza. Aporta 22 títulos de 6 autores.

- 1.000 millones: poesía en lengua española del siglo XXI, editada por Daniel Helder, Daiana Henderson y Bernardo Orge aporta una lista que reúne a 33 autores hispanoamericanos nacidos entre 1980 y 1995 y cuyas obras fueron editadas previamente en soporte digital o en soporte papel. La antología aporta una serie de propuestas poéticas desarrolladas en Latinoamérica, Europa y EE.UU. durante la última década. Aporta 95 títulos de 32 autores.

- Bogotáz9 es el resultado de una selección realizada por los escritores colombianos Piedad Bonnet, Héctor Abad Faciolince y Óscar Collazos. El listado de escritores elaborado se basó en la nominación de más de 2 mil editores, críticos y lectores de todo el continente. Aporta 133 títulos de 36 autores. 
- La gran novela latinoamericana, de Carlos Fuentes, aporta un repertorio de autores y novelas desde la perspectiva de un autor del "boom". Aporta 124 títulos de 65 autores.

- Una sección de títulos de escritores caribeños, antillanos y de las Guayanas diseñada sobre la base de la combinación de los títulos y escritores que componen la compilación West Indian Narrative: An Introductory Anthology (Ramchand); la antología They Came in Ships: An Anthology of Indo-Guyanese Writing (Benjamin, Kallicharan, McDonald y Searwar); la selección The Routledge Reader in Caribbean Literature (Donnell y Lawson Welsh); The Companion to Anglophone Caribbean Literature (Donnel y Bucknor); la selección The Oxford Book of Caribbean Verse (Brown y McWatt) y los escritores caribeños, antillanos y de las Guayanas de la Encyclopedia of Latin American and Caribbean Literature 1900-2003, editada por Daniel Balderston y Mike Gonzalez. Aporta 2.597 títulos de 559 autores.

Si el Diccionario elaborado por Aira propone autores y obras en español y portugués, Los chongos de Roa Bastos. Narrativa contemporánea de Paraguay y Saraus. Movimiento - Literatura - Periferia - São Paulo aportan en un caso obras en jorapa, guaraní y distintas formas de heteroglosia e hibridaciones entre guaraní, castellano y otras lenguas y hablas indígenas $y$, en otro caso, textos en el portugués de los jóvenes de la perifatividade paulista. Aporta 33 títulos de 9 autores.

Además, se elaboró un segmento específico para que la literatura precolombina esté representada en el CLLyC. Este segmento construido aporta 50 títulos de literaturas precolombinas y ocho autores (muchas de las obras son anónimas o colectivas). A su vez, si Saraus. Movimiento Literatura - Periferia - São Paulo aporta títulos de autores de las nuevas formas textuales, los diccionarios de Aira y Cella, la enciclopedia Latin American Women Writers, el ensayo de Latin American Writers on Gay and Lesbian Themes: A Bio-Critical Sourcebook, y la Encyclopedia of Latin American and Caribbean Literature 1900-2003, aportan también títulos de las formas narrativas tradicionales de Brasil.

Latin American Women Writers: An Encyclopedia aporta obras de 258 escritoras nacidas entre los siglos XVI y fines del siglo XX. Los chongos de Roa Bastos. Narrativa contemporánea de Paraguay y Saraus. Movimiento Literatura - Periferia - São Paulo contribuyen con obras de autores nacidos en el periodo de 1965 y 1984 en el primer caso, De la Tricolor a la Wiphala. Narrativa contemporánea de Bolivia propone autores nacidos entre 1956 
y mediados de los años 1980; en el caso de Saraus, aporta autores nacidos entre 1957 y mediados de los años 1980 que en su mayoría inician sus trabajos literarios en las décadas del 1990 y principios de los 2000; así como McOndo (una antología de la nueva literatura hispanoamericana) aporta escritores nacidos entre 1959 y que se destacan en su trabajo literario a mediados de los años 1990. La antología 1.00o millones: poesía en lengua española del siglo XXI incluye 33 autores hispanoamericanos nacidos entre 1980 y 1995 y el Manifiesto del Crack incorpora 6 autores mexicanos nacidos entre 1961 y 1968 y cuyas obras son todas posteriores a 1996. Asimismo, la selección de Bogotá39 menciona 39 autores menores de 39 años en el año 2007.

Si el Manifiesto del Crack propone obras que "faciliten su traducción" (Santos, 2013, p. 288), Los chongos de Roa Bastos y Saraus aportan textos con complejidades textuales de difícil traducción habida cuenta de las distintas formas de utilización de heteroglosia y hablas coloquiales y registros y variedades dialectales tanto del castellano como del guaraní, del portugués y del jorapa, y otras lenguas y hablas indígenas y, por otro lado, las formas del habla del portugués de los jóvenes de los barrios periféricos de São Paulo. De la Tricolor a la Wiphala. Narrativa contemporánea de Bolivia contribuye con textos escritos mayoritariamente en las distintas variedades del castellano de Bolivia, excepto en el caso Alison Spedding, que escribe también en aymará. El ensayo de Latin American Writers on Gay and Lesbian Themes: A Bio-Critical Sourcebook de David Foster Wallace colabora con textos escritos en español y en portugués, aunque el autor trabaja con obras en español y portugués y obras traducidas al inglés. La selección de Bogotá39 incluye autores que escriben en español, portugués e inglés.

Si los cuentos elegidos para $\mathrm{McOndo}$ promulgan una postura narrativa rupturista con la del realismo mágico latinoamericano, Carlos Fuentes en La gran novela latinoamericana nos brinda la perspectiva de abordaje de un escritor del boom, que considera que en Cien años de soledad, de Gabriel García Márquez, se encuentra "la tensión entre Utopía, Epopeya y Mito" (FUENTES, 2014, p. 262), cuyo tiempo es la simultaneidad, donde "los hombres se defienden con la imaginación del caos circundante, de las selvas y los ríos, del inmenso, devorador magma suramericano" (FUENTES, 2014, p. 265).

Mientras la mayoría de las fuentes utilizadas no destaca una perspectiva de género (cosa que sí pueden realizar específicamente 
los autores mencionados al interior de sus textos), el repertorio Latin American Women Writers: An Encyclopedia, editado por María André y Eva Bueno (2008), nos aporta un registro de distintas formas narrativas escrito por escritoras latinoamericanas, con una estrategia de abordaje teórico de literatura latinoamericana femenina. Aporta 2.951 títulos de 303 autoras. Otro tanto agrega el ensayo de Latin American Writers on Gay and Lesbian Themes: A Bio-Critical Sourcebook de David Foster Wallace: propone 27 títulos de 25 autores latinoamericanos en todos los géneros, con una estrategia que guía la incorporación de estos autores y obras que es la de incluir en el diseño del CLLyC la perspectiva que articula la relación entre homosexualidad y sociedad en la literatura latinoamericana.

Si McOndo y De la Tricolor a la Wiphala proponen cuentos, el Manifiesto del Crack propone novelas, al igual que la propuesta de Carlos Fuentes en la Gran novela latinoamericana; Saraus propone poesías, al igual que la antología 1.000 millones, aunque en este caso, algunos de los autores también escriben novelas, cuentos, cómics (que fueron incorporados al diseño del CLLyC).

El segmento de títulos de escritores caribeños, antillanos y de las Guayanas diseñado sobre la base de la combinación de los títulos y escritores que componen los trabajos mencionados previamente contribuye con 559 autores y más de 2.597 obras, con un criterio de amplitud en la incorporación de autores: dramaturgos, poetas, novelistas, cuentistas, científicos, historiadores, cronistas, periodistas y ensayistas. La radicalidad de esta incorporación se enmarca en una concepción de articulación de "lo caribeño" como unidad, que "siendo unidad, no es inseparable de América Latina” (PIZARRO, 1987, p. 146).

En el caso del Catálogo de Editoriales Cartoneras latinoamericanas de la Universidad de Wisconsin, la incorporación en el CLLyC de sus títulos y autores introduce una perspectiva de inscripción vertical, es decir, su inscripción atraviesa todas las perspectivas poéticas y consideraciones que hemos descrito previamente. Asimismo, la incorporación de los títulos y autores de las editoriales cartoneras ${ }^{2}$ nos provee de títulos para la construcción de un CLLyC que contenga, asimismo, formas editoriales que integran la dispositividad material de la galaxia Gutenberg, pero que

${ }^{2}$ Las editoriales cartoneras se caracterizan tanto por emplear formatos artesanales como tapas y diseños coloreados libro por libro, como por la impresión de tapas en cartón y cartón corrugado e interiores mayoritariamente fotocopiados. 
se constituyen como artefactos culturales propios de la oferta específica de las literaturas latinoamericanas. Aporta 469 títulos de 358 autores.

En el caso de Encyclopedia of Latin American and Caribbean Literature1900-2003, editada por Daniel Balderston y Mike Gonzalez (2004), se separaron los autores caribeños, antillanos y de las Guayanas y se los incorporó a la sección de escritores caribeños, antillanos y de las Guayanas del CLLyC. Los restantes autores fueron incorporados como otra sección de las búsquedas. Aporta 2.672 títulos de 651 autores.

- Lenguas de las obras: las distintas variedades del castellano y del portugués de América Latina, francés, inglés, neerlandés, guaraní, náhuatl, aymará, tomáraho, ashlushlay, ybytozo, toba quom, sanapaná, maká, axe-guayaki, ayoreo quiché, patois, créole, jorapa, portuñol y heteroglosias de la región.

- Se relevaron autores de 43 nacionalidades. ${ }^{3}$

La presencia de autores de nacionalidad española y portuguesa se debe a dos tipos de inclusiones: por un lado, son autores (cronistas, historiadores o directamente "conquistadores") de la etapa de la "Conquista" y, por el otro, son autores incluidos en la antología 1.000 millones, poesía en lengua española del siglo XXI, y en La gran novela latinoamericana, de Carlos Fuentes. Asimismo, el CLLyC incorpora autores nacidos en EE.UU. y que se radicaron en América Latina o poseen ciudadanía de alguno de los países de América Latina y el Caribe y autores nacidos en EE.UU. y que producen literatura latinoamericana y caribeña desde ese país.

En algunos casos, se rastrearon títulos de los autores por fuera de los mencionados en esas fuentes y se incorporaron al CLLyC en función de que la muestra pudiese efectuarse por sus libros y no por poemas separados o cuentos, relatos y pequeños ensayos sueltos.

En todos los casos se realizó la búsqueda de los títulos en impreso y en digital. La obtención de datos en ambos soportes facilita la comparación de la transposición de los títulos.

Se sintetiza: 20.409 títulos diferentes de 2.932 autores diferentes.

${ }^{3}$ 1. Argentina, 2. Bolivia, 3. Brasil, 4. Chile, 5. Colombia, 6. Costa Rica, 7. Cuba, 8. Ecuador, 9. El Salvador, 10. Guatemala, 11. Honduras, 12. México, 13. Nicaragua, 14. Panamá, 15. Paraguay, 16. Perú, 17. Puerto Rico, 18. Republica Dominicana, 19. Uruguay, 20. Venezuela, 21. Trinidady Tobago, 22. Islas Vírgenes, 23. San Vicentey las Granadinas, 24. Santa Lucía, 25. Martinica, 26. Jamaica, 27. Surinam, 28. Guyana, 29. Dominica, 30. Barbados, 31.Saint Maarten, 32. Haití, 33. Bahamas, 34. Grenada, 35. Guadalupe, 36. Belice, 37. Montserrat, 38. Antigua y Barbuda, 39. Aruba, 40.San Cristóbaly Nieves (Saint Kittsand Nevis), 41. España, 42. Portugal, 43. EE.UU. 


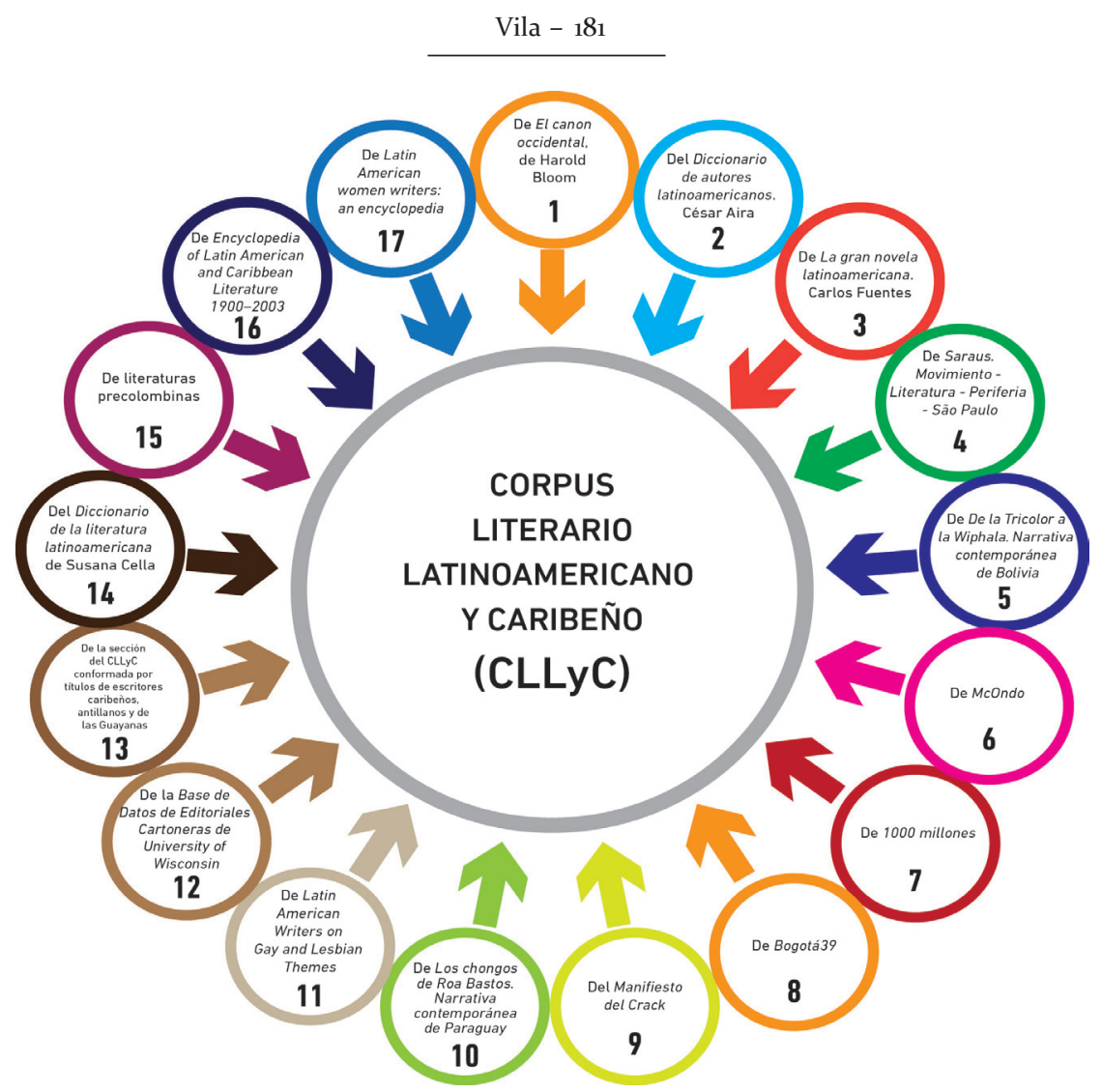

Figura 1. Composición del CLLyC. Elaboración propia.

\section{DEFINICIÓN DE PLATAFORMAS COMERCIALES Y BIBLIOTECAS EN DONDE SE REALIZARÁN LAS BÚSQUEDAS. ¿DÓNDE BUSCAR?}

Se seleccionaron 9 bibliotecas digitales y 14 plataformas comercializadoras en digital y servicios de lectura en streaming y descarga para realizar el estudio comparativo de la disponibilidad de los títulos de los autores latinoamericanos y caribeños del CLLyC.

- Criterios destacados para la selección de las plataformas y bibliotecas y características de ellas:

- Amazon dispone de 1.700.ooo títulos de libros electrónicos en su catálogo y posee una posición dominante en el mercado de EE.UU. (MILLIOT, 2011; WISCHENBART et al., 2013, p. 18). Su servicio de suscripción por tarifa plana Kindle Unlimited posee un catálogo de 700 mil títulos. 
- Barnes \& Noble dispone casi 3 millones de títulos de libros digitales con cerca del 27\% de los compradores de libros electrónicos (MILLIOT, 2011).

- iBookstore y iTunes. En junio de 2011, Apple indicó que vendió 130 millones de libros electrónicos a través de iTunes. En octubre de 2012, vendió 400 millones. La aplicación iBooks (en 30 idiomas) es la residente en las tabletas iPad.

- Google Books ofrece sus servicios de casi 350 mil títulos combinando sus ventas directas con el formato similar al de un distribuidor mayorista tradicional.

- Kobo es una plataforma canadiense de libros electrónicos de estándares abiertos, presente en 190 países, que alcanza a 10 millones de usuarios y posee un catálogo de más de 4 millones de libros, en 68 idiomas y un destacado e-reader.

- Casa del Libro ofrece un catálogo de alrededor de 1 millón de títulos, con aproximadamente 1 millón de usuarios registrados y 118 tiendas en España.

- Las búsquedas en Free-Ebooks se realizaron como forma de establecer también un estado del arte en plataformas de descargas gratuitas de libros digitales.

- Gandhi es la principal librería en físico y en digital de México; Livraria Cultura es la principal librería en físico y digital de Brasil. Dispone de un catálogo de 8 millones de títulos en soporte papel y cerca de 1 millón de títulos en digital; Perubookstore.com es la principal librería online de Perú; y Librería Santa Fe es la principal plataforma comercial de libros en digital ( 5 mil títulos) de Argentina.

- También se efectuaron búsquedas en plataformas que representan "nuevos modelos" de venta: Nubico y Scribd: Nubico es una plataforma digital de venta por suscripción. Cuenta con 100 mil clientes y dispone de 7.700 títulos de 83 editoriales. Scribd es una aplicación online en la que se pueden subir documentos y presentaciones para poder mostrarlas online con opciones de descarga. Posee un catálogo de 500 mil libros a los que se puede acceder por "tarifa plana".

- Todas las búsquedas se efectuaron en las bibliotecas más significativas desde el punto de vista lingüístico y que se destacan por prestaciones, arquitectura y disponibilidad digital (CHOWDURY; FOO, 2012; KLIBANSKI, 2006; TRAMULLAS SAZ, 2002).

- Public Library New York y Columbia University Libraries forman parte del grupo de las bibliotecas con mayor prestigio a nivel internacional por la calidad de los recursos que ofrecen (y por reconocimiento en el campo académico).

- La Biblioteca Nacional de España es la biblioteca más reconocida en el campo idiomático del español, así como Gallica - Biblioteca Nacional de Francia es la biblioteca más reconocida internacionalmente en lengua francesa y Europeana, a escala continental europea. 
- La Biblioteca Nacional de Perú y la Biblioteca de São Paulo se seleccionaron con el criterio de incorporar las más reconocidas bibliotecas latinoamericanas con colecciones en digital.

- La Biblioteca del Instituto Latinoamericano de Berlín se seleccionó como biblioteca especializada en temas y autores latinoamericanos.

\section{BÚSQUEDAS EN SO PORTE IMPRESO Y EN DIGITAL DE LOS TÍTULOS Y AUTORES DEL CORPUS EN LAS PLATA- FORMAS COMERCIALES Y BIBLIOTECAS DEFINIDAS Y RE- COLECCIÓN DE DATOS. ¿QUÉ RESULTADOS NOS PERMI- TE OBTENER EL DISEÑO DEL CORPUS?}

\section{Acciones:}

- Cruce de datos de las búsquedas en plataformas y en bibliotecas para la localización de coincidencias mediante la construcción de un "bot" para recolectar los resultados de la búsqueda de cada obra y autor del CLLyC en las diferentes plataformas. El mecanismo de búsqueda consistió en la carga de autor + título + fuente del CLLyC; búsqueda de autores del CLLyC en c/plataforma y biblioteca; verificación de resultados por criterios de coincidencia (exacta + flexible + similitud laxa).

- Identificación de obras y autores del CLLyC que han sido traspuestas a digital y detalle de cada obra en soporte papel/digital. Tabla 1).

- Creación de tablas con datos recolectados (con los campos que observamos en

La base de datos almacena 2.262.364 registros de resultados (por tuplas: compuestas por: sitio + libro buscado + autor buscado + título encontrado + soporte + autor encontrado).

Todas las búsquedas se hicieron en el idioma original de las obras.

Se realizaron 61.551 búsquedas solo de autores.

Se realizaron 207.570 búsquedas de autor + libro. 
184 - Remate de Males 36.1

\subsection{Algunos resultados por Plataforma/Biblioteca}

\begin{tabular}{|c|c|c|c|c|c|c|c|c|c|c|c|c|}
\hline Plataforma / Biblioteca & $\begin{array}{l}\text { Autores } \\
\text { Impreso }\end{array}$ & $\begin{array}{l}\text { Autores } \\
\text { Unicos } \\
\text { Impreso }\end{array}$ & $\begin{array}{l}\text { \% Autores } \\
\text { Unicos } \\
\text { Impreso }\end{array}$ & $\begin{array}{l}\text { Títulos } \\
\text { Impreso }\end{array}$ & $\begin{array}{l}\text { Títulos } \\
\text { Únicos } \\
\text { Impreso }\end{array}$ & $\begin{array}{c}\text { \% Titulos } \\
\text { Unicos } \\
\text { Impreso }\end{array}$ & $\begin{array}{c}\text { Autores } \\
\text { Digital }\end{array}$ & $\begin{array}{l}\text { Autores } \\
\text { Unicos } \\
\text { Digital }\end{array}$ & $\begin{array}{l}\text { \% Autores } \\
\text { Unicos } \\
\text { Digital }\end{array}$ & $\begin{array}{l}\text { Títulos } \\
\text { Digital }\end{array}$ & $\begin{array}{l}\text { Títulos } \\
\text { Unicos } \\
\text { Digital }\end{array}$ & $\begin{array}{l}\text { \% Titulos } \\
\text { Unicos } \\
\text { Digital }\end{array}$ \\
\hline Amazon & 1728 & 35 & $2.03 \%$ & 7144 & 485 & $6.79 \%$ & 432 & 31 & $7.18 \%$ & 1101 & 119 & $10.81 \%$ \\
\hline Barnes \& Noble & 358 & 2 & $0.56 \%$ & 795 & 23 & $2.89 \%$ & 250 & 6 & $2.4 \%$ & 600 & 48 & $8 \%$ \\
\hline Biblioteca de São Paulo & 78 & 0 & $0 \%$ & 218 & 2 & $0.92 \%$ & 0 & 0 & $0 \%$ & 0 & 0 & $0 \%$ \\
\hline Biblioteca I. Lat. Berlín & 1479 & 24 & $1.62 \%$ & 4865 & 198 & $4.07 \%$ & 175 & 27 & $15.43 \%$ & 319 & 85 & $26.65 \%$ \\
\hline Biblioteca D. Unión Europea & 0 & 0 & $0 \%$ & 0 & 0 & $0 \%$ & 281 & 31 & $11.03 \%$ & 582 & 0 & $0 \%$ \\
\hline Biblioteca Nacional de España & 727 & 0 & $0 \%$ & 2473 & 40 & $1.62 \%$ & 522 & 172 & $32.95 \%$ & 1749 & 1137 & $65.01 \%$ \\
\hline Biblioteca Nacional de Francia & 1186 & 9 & $0.76 \%$ & 3685 & 89 & $2.42 \%$ & 20 & 3 & $15 \%$ & 23 & 9 & $39.13 \%$ \\
\hline Biblioteca Nacional de Perú & 913 & 4 & $0.44 \%$ & 2855 & 208 & $7.29 \%$ & 9 & 2 & $22.22 \%$ & 10 & 0 & $0 \%$ \\
\hline Casa del Libro & 729 & 1 & $0.14 \%$ & 2211 & 30 & $1.36 \%$ & 198 & 0 & $0 \%$ & 512 & 5 & $0.98 \%$ \\
\hline Columbia University Libraries & 1257 & 4 & $0.32 \%$ & 4663 & 88 & $1.89 \%$ & 275 & 0 & $0 \%$ & 572 & 164 & $28.67 \%$ \\
\hline Free E-Books & 0 & 0 & $0 \%$ & 0 & 0 & $0 \%$ & 45 & 0 & $0 \%$ & 88 & 7 & $7.95 \%$ \\
\hline Gandhi & 231 & 0 & $0 \%$ & 666 & 4 & $0.6 \%$ & 240 & 3 & $1.25 \%$ & 617 & 10 & $1.62 \%$ \\
\hline Google Books & 2230 & 173 & $7.76 \%$ & 11140 & 1877 & $16.85 \%$ & 92 & 12 & $13.04 \%$ & 143 & 37 & $25.87 \%$ \\
\hline iBook Store & 0 & 0 & $0 \%$ & 0 & 0 & $0 \%$ & 173 & 1 & $0.58 \%$ & 368 & 10 & $2.72 \%$ \\
\hline Kobo & 0 & 0 & $0 \%$ & 0 & 0 & $0 \%$ & 123 & 5 & $4.07 \%$ & 262 & 26 & $9.92 \%$ \\
\hline Librería Santa Fe & 548 & 0 & $0 \%$ & 1890 & 69 & $3.65 \%$ & 150 & 2 & $1.33 \%$ & 336 & 20 & $5.95 \%$ \\
\hline Livraria Cultura & 534 & 0 & $0 \%$ & 1785 & 103 & $5.77 \%$ & 337 & 21 & $6.23 \%$ & 1035 & 185 & $17.87 \%$ \\
\hline Nubico & 0 & 0 & $0 \%$ & 0 & 0 & $0 \%$ & 182 & 0 & $0 \%$ & 496 & 1 & $0.2 \%$ \\
\hline Perú Book Store & 36 & 0 & $0 \%$ & 76 & 3 & $3.95 \%$ & 45 & 1 & $2.22 \%$ & 154 & 2 & $1.3 \%$ \\
\hline Public Library New York & 1297 & 4 & $0.31 \%$ & 5760 & 171 & $2.97 \%$ & 8 & 0 & $0 \%$ & 21 & 0 & $0 \%$ \\
\hline Scribd & 0 & 0 & $0 \%$ & 0 & 0 & $0 \%$ & 715 & 209 & $29.23 \%$ & 1718 & 864 & $50.29 \%$ \\
\hline
\end{tabular}

Tabla 1. Comparación de resultados de las ofertas en papel / digital de los títulos y autores del CLLyC por plataforma y biblioteca. Elaboración propia.

Comovemos en la Tabla 1, en relación con los títulos únicos en soporte impreso por plataforma y biblioteca (títulos en soporte papel que solo están disponibles en esa plataforma o biblioteca) destacamos una oferta mediana en Google Books y Amazon: ofrecen 1.877 títulos únicos sobre 11.140 títulos y 485 títulos únicos sobre 7.144 títulos, respectivamente. Livraria Cultura, de São Paulo, dispone de 1.785 (12,03\%), Gandhi (México) ofrece 666 títulos (4,48\%). Perubookstore ofrece 76 títulos (o,51\% del total).

En soporte digital, la plataforma que más títulos ofrece es la Web de intercambios Scribd (esto ratifica que la participación del usuario en la oferta, produce un incremento de las posibilidades de acceso): 1.718 títulos sobre 6.447 títulos hallados en digital, es decir, el 35,48\% de los títulos existentes en digital para las búsquedas efectuadas. Livraria Cultura ofrece 1.035 (21,37\% de los títulos disponibles en digital); Barnes \& Noble, 600 títulos en digital (12,39\%). En la mayoría de las plataformas comercializadoras, autores y títulos del corpus propuesto gozan de visibilidad y oferta en soporte papel, pero el proceso de transposición a digital es lento.

En relación con la oferta en soporte impreso en bibliotecas, la biblioteca que más títulos dispone es la Public Library of New York, con 
$5 \cdot 760$ (el $38,82 \%$ de la oferta en soporte papel). Luego la siguen la Biblioteca del Instituto Latinoamericano de Berlín con 4.865 títulos (32,79\% del total de los títulos de la búsqueda) y Columbia University Libraries con 4.663 títulos en soporte papel (31,43\%). De las demás bibliotecas analizadas, se destaca, además, la oferta en soporte impreso de la Biblioteca Nacional de Francia: 3.685 títulos (24,83\%) y de la Biblioteca Nacional de Perú: 2.855 títulos (19,24\%). La plataforma Google Books dispone de $\mathbf{2 . 2 3 0}$ autores en soporte impreso (el 90,02\% del total de autores relevados en soporte papel). La Biblioteca del Instituto Latinoamericano de Berlín dispone de 1.479 autores en soporte impreso (el 59,70\% de los autores disponibles en impreso); Public Library New York dispone de 1.297 autores en soporte impreso (el 52,36\% de los autores latinoamericanos y caribeños disponibles en impreso). La Biblioteca Nacional de Perú se destaca entre las latinoamericanas: ofrece 913 autores en soporte impreso (el 36,85\%).

En relación con la oferta en digital en bibliotecas, la biblioteca que más títulos ofrece es la Biblioteca Nacional de España: 1.749 (el 36,12\% de la oferta disponible en digital). Este dato es muy destacable, dado que la Biblioteca Nacional de España ofrece en digital más títulos que las demás plataformas y bibliotecas en las que se efectuaron las búsquedas (incluida la Web de intercambio Scribd).

En relación con los títulos únicos en digital por plataforma y biblioteca (títulos en digital que solo están disponibles en esa plataforma o biblioteca) la oferta de Biblioteca Nacional de España es muy destacada: dispone de 1.137 títulos únicos sobre 1.749 títulos que ofrece en total en soporte digital. Luego, la Web de intercambio Scribd dispone de 864 títulos únicos sobre 1.718 títulos que ofrece en digital. Livraria Cultura ofrece 185 títulos únicos sobre 1.035 títulos del total en digital. 
186 - Remate de Males 36.1

\subsection{Algunos resultados por títulos y autores}

\begin{tabular}{|c|c|c|c|}
\hline Autor & Obra & Fuentes & Veces \\
\hline Carpentier, Alejo & Concierto Barroco & $2,13,14$ & 14 \\
\hline Carpentier, Alejo & Los pasos perdidos & $1,2,3,13,14$ & 14 \\
\hline Vargas Llosa, Mario & Los cachorros & $2,3,14$ & 14 \\
\hline Allende, Isabel & La casa de los espíritus & 3 & 13 \\
\hline Arlt, Roberto & El juguete rabioso & 2 & 13 \\
\hline Azuela, Mariano & Los de abajo & $2,3,14$ & 13 \\
\hline Benedetti, Mario & Inventario & 2,14 & 13 \\
\hline Bolaño, Roberto & Los detectives salvajes & 6 & 13 \\
\hline Borges, Jorge Luis & El Aleph & $1,2,3,14$ & 13 \\
\hline Cambaceres, Eugenio & Sin rumbo & 2,14 & 13 \\
\hline Carpentier, Alejo & El reino de este mundo & $1,2,3,13,14$ & 13 \\
\hline Neruda, Pablo & Veinte poemas de amor y una canción desesperada & $1,2,14$ & 13 \\
\hline Roncagliolo, Santiago & Abril rojo & 3,8 & 13 \\
\hline Rulfo, Juan & Pedro Páramo & $2,3,14$ & 13 \\
\hline Alegría, Ciro & El mundo es ancho y ajeno & 2,14 & 12 \\
\hline Altamirano, Ignacio Manuel & Clemencia & 2 & 12 \\
\hline Altamirano, Ignacio Manuel & El Zarco & 2,14 & 12 \\
\hline Amado, Jorge & Gabriela, cravo e canela & 2,14 & 12 \\
\hline Amado, Jorge & Sudor & 2 & 12 \\
\hline Arguedas, Alcides & Raza de bronce & 2,14 & 12 \\
\hline Arguedas, José María & Los ríos profundos & 2,14 & 12 \\
\hline Arguedas, José María & Yanar fiesta & 2 & 12 \\
\hline Arlt, Roberto & Aguafuertes porteñas & 2 & 12 \\
\hline Arlt, Roberto & Los lanzallamas & 2 & 12 \\
\hline Arlt, Roberto & Los siete locos & 2 & 12 \\
\hline Asturias, Miguel Angel & El señor Presidente & 2,14 & 12 \\
\hline Asturias, Miguel Angel & Hombres de maíz & $1,2,14$ & 12 \\
\hline Asturias, Miguel Ángel & Leyendas de Guatemala & 2,14 & 12 \\
\hline Benedetti, Mario & La borra del café & 14 & 12 \\
\hline Benedetti, Mario & La muerte y otras sorpresas & 2,14 & 12 \\
\hline Benedetti, Mario & La tregua & 2,14 & 12 \\
\hline Benedetti, Mario & Pedro y el capitán & 14 & 12 \\
\hline Benedetti, Mario & Primavera con una esquina rota & 2 & 12 \\
\hline Bioy Casares, Adolfo & Historias de amor & 2 & 12 \\
\hline Borges, Jorge Luis & Ficciones & $1,2,3,14$ & 12 \\
\hline Borges, Jorge Luis & Inquisiciones & 2,14 & 12 \\
\hline Borges, Jorge Luis & Obras completas & 2,14 & 12 \\
\hline Cambaceres, Eugenio & En la sangre & 2,14 & 12 \\
\hline Cané, Miguel & Juvenilia & 2,14 & 12 \\
\hline Carpentier, Alejo & El acoso & $2,13,14$ & 12 \\
\hline Carpentier, Alejo & El recurso del método & $1,2,3,13,14$ & 12 \\
\hline Castellanos, Rosario & Oficio de tinieblas & 2,14 & 12 \\
\hline Conti, Haroldo & Sudeste & 2,14 & 12 \\
\hline Cortázar, Julio & Bestiario & 2,3 & 12 \\
\hline Cortázar, Julio & Las armas secretas & 2,14 & 12 \\
\hline Cortázar, Julio & Octaedro & 2,14 & 12 \\
\hline Donoso, José & El Mocho & 2,14 & 12 \\
\hline Filloy, Juan & Op Oloop & 2,14 & 12 \\
\hline Neruda, Pablo & Canto general & $1,2,14$ & 12 \\
\hline Neruda, Pablo & Residencia en la tierra & $1,2,14$ & 12 \\
\hline
\end{tabular}

Tabla 2. Los 50 títulos del corpus que más aparecen en soporte impreso. Elaboración propia.

En el nivel de la oferta de títulos en impreso (ver Tabla 2) de 14.835 títulos relevados en las búsquedas en 14 plataformas y bibliotecas que disponen de títulos en impreso, solo 3 títulos son ofrecidos en todas las plataformas y bibliotecas: 2 títulos del escritor cubano Alejo Carpentier (Los pasos perdidos; Concierto barroco) y 1 título de Mario Vargas Llosa (Los cachorros). De los títulos que los siguen, 11 obras fueron relevadas en 13 plataformas y bibliotecas, de los cuales 4 títulos pertenecen al 
canon de Bloom: El Aleph, de Jorge Luis Borges; Pedro Páramo, de Juan Rulfo; El reino de este mundo, de Alejo Carpentier y 20 poemas de amor y una canción desesperada, de Pablo Neruda. En este alto segmento marcamos que se encuentra la obra de Roberto Bolaño, Los detectives salvajes. Culminan la élite de la oferta en títulos las obras de autores canónicos Miguel Ángel Asturias (Leyendas de Guatemala y Hombres de maíz) y de obras tradicionales o relacionadas al folklore de algunos países latinoamericanos, en dominio público, de Miguel Arguedas (Los ríos profundos); Mariano Azuela (Los de abajo); Eugenio Cambaceres (Sin rumbo) y Ricardo Palma (Tradiciones), y el clásico de Roberto Arlt El juguete rabioso, en dominio público. Finalmente, se destacan Gabriela, cravo e canela, de Jorge Amado, Inventario, de Mario Benedetti y el bestseller de Isabel Allende, La casa de los espíritus.

\begin{tabular}{|c|c|c|c|c|}
\hline Autor & Obra & Fuentes & & $=$ \\
\hline Cieza de León, Pedro & Crónica del Perú & 2 & 14 & 09 \\
\hline Mármol, José & Amalia & 2 & 14 & 10 \\
\hline Alberdi, Juan Bautista & Bases y puntos de partida para la organización política... & 2,14 & 13 & 09 \\
\hline Alberdi, Juan Bautista & El crimen de la guerra & 2,14 & 13 & 11 \\
\hline Altamirano, Ignacio Manuel & Clemencia & 2 & 13 & 12 \\
\hline Benedetti, Mario & La tregua & 2,14 & 13 & 12 \\
\hline Darío, Rubén & Prosas profanas & 2,3 & 13 & 09 \\
\hline Lillo, Baldomero & Sub Terra & 2,14 & 13 & 08 \\
\hline Villaverde, Cirilo & Cecilia Valdés & 2,14 & 13 & 12 \\
\hline Acevedo Díaz, Eduardo & Brenda & 14 & 12 & 04 \\
\hline Barros Arana, Diego & Historia general de Chile & 2,14 & 12 & 08 \\
\hline Blest Gana, Alberto & Martín Rivas & 14 & 12 & 10 \\
\hline Borges, Jorge Luis & El Aleph & $1,2,3,14$ & 12 & 13 \\
\hline Cruz, Sor Juana Inés de la & Obras & 2 & 12 & 11 \\
\hline Darío, Rubén & Cantos de vida y de esperanza & $2,3,14$ & 12 & 10 \\
\hline Ercilla y Zúñiga, Alonso de & La araucana & 2 & 12 & 09 \\
\hline Garcilaso de la Vega & Comentarios reales & 2 & 12 & 10 \\
\hline Ingenieros, José & El hombre mediocre & 2,14 & 12 & 10 \\
\hline Lillo, Baldomero & Sub sole & 2 & 12 & 08 \\
\hline López de Gómara, Francisco & Historia general de las Indias & 2 & 12 & 10 \\
\hline Rodó, José Enrique & Ariel & 2,14 & 12 & 10 \\
\hline Aguinis, Marcos & La gesta del marrano & 14 & 11 & 08 \\
\hline Asturias, Miguel Ángel & El señor Presidente & 2,14 & 11 & 12 \\
\hline Borges, Jorge Luis & Ficciones & $1,2,3,14$ & 11 & 12 \\
\hline Borges, Jorge Luis & Historia universal de la infamia & $2,3,14$ & 11 & 11 \\
\hline Cané, Miguel & Juvenilia & 2,14 & 11 & 12 \\
\hline Cieza de León, Pedro & Señorío de los Incas & 2 & 11 & 08 \\
\hline Dario, Rubén & Canto errante & & 11 & 06 \\
\hline Darío, Rubén & Tierras solares & 2,14 & 11 & 07 \\
\hline Diaz del Castillo, Bernal & Historia verdadera de la conquista de la Nueva España & 2 & 11 & 11 \\
\hline García Márquez, Gabriel & La hojarasca & 2,14 & 11 & 08 \\
\hline García Márquez, Gabriel & La Mala Hora & 2,14 & 11 & 08 \\
\hline Güiraldes, Ricardo & Don Segundo Sombra & 2,14 & 11 & 08 \\
\hline Las Casas, Bartolomé de & Historia de las Indias & 2 & 11 & 06 \\
\hline Lugones, Leopoldo & Las fuerzas extrañas & 2,14 & 11 & 09 \\
\hline Montenegro, Carlos & Hombres sin mujer & 2,13 & 11 & 05 \\
\hline Neruda, Pablo & Canto general & $1,2,14$ & 11 & 12 \\
\hline Nervo, Amado & Los jardines interiores & 2,14 & 11 & 06 \\
\hline Nervo, Amado & Misticas & 2 & 11 & 07 \\
\hline Palma, Ricardo & Tradiciones & 2 & 11 & 12 \\
\hline Palma, Ricardo & Tradiciones peruanas & 2,14 & 11 & 12 \\
\hline Rabasa, Emilio & La bola & 2 & 11 & 09 \\
\hline Altamirano, Ignacio Manuel & ElZarco & 2,14 & 10 & 12 \\
\hline Altamirano, Ignacio Manuel & La Navidad en las montañas & 2,14 & 10 & 11 \\
\hline Arlt, Roberto & El juguete rabioso & 2 & 10 & 13 \\
\hline Borges, Jorge Luis & El Hacedor & $1,2,14$ & 10 & 10 \\
\hline Cané, Miguel & El viaje & 2 & 10 & 11 \\
\hline Carriego, Evaristo & Misas herejes & 2,14 & 10 & 10 \\
\hline Cervantes de Salazar, Francisco & Crónica de Nueva España & 2 & 10 & 08 \\
\hline Cortázar, Julio & Final del juego & 2 & 10 & 09 \\
\hline
\end{tabular}

Tabla 3. Los 50 títulos que más aparecen en digital. Elaboración propia. 
En una orientación de los resultados más destacados de las búsquedas de las obras y autores del CLLyC diseñado efectuadas, subrayamos especialmente que los resultados de los 50 títulos del CLLyC que más aparecen en soporte digital (ver Tabla 3 ) nos exhiben que los títulos cuyos derechos de autor están en dominio público encabezan la transposición a digital de la literatura latinoamericana y caribeña en impreso. Sin embargo, de los primeros 50 títulos que aparecen en soporte digital en las búsquedas realizadas (aquellos que aparecen 14, 13, 12 y 11 veces en la misma cantidad de plataformas y bibliotecas), 41 son títulos de autores cuyas obras están en dominio público. Esto ratifica uno de los criterios de transposición por parte de la industria: se transpone con mayor frecuencia aquello por lo cual no se cotizan derechos de autor. A su vez, aparece un lote de algunos de los títulos bloomianos en la élite de la oferta. De los primeros 50 títulos, 12 títulos son de autores bloomianos y 3 de ellos son títulos bloomianos. Este listado superior, es decir, el segmento que aparece con mayor recurrencia en las tablas de resultados de las búsquedas de la investigación aparece como aportado desde estrategias editoriales y bibliotecológicas acompañadas por acciones relacionadas con la inclusión de títulos mediante ventanas al gusto de los lectores.

Igualmente, la intervención de los usuarios en Scribd funciona como ventana por la cual los criterios poéticos, estéticos, sobre la literatura latinoamericana y caribeña ingresarían en las formas de construcción del catálogo general en digital: de los 6.447 títulos hallados en digital, Scribd ofrece 1.718 títulos, es decir, el 35,48\% de los títulos existentes en digital para las búsquedas efectuadas. Además, como otra ventana al gusto de los lectores, el modelo de adquisición bibliotecaria impulsada por el usuario (Patron Driven Acquisition-PDA) se constituye en un cambio paradigmático, en el que la "autoridad bibliotecaria" deja espacio a participación directa del usuario en la construcción de la colección: alrededor de 600 bibliotecas universitarias de todo el mundo articulan sus fondos bibliográficos desde esta estrategia (ESPOSITO et al., 2012, web).

Así, se deduce de los resultados de las búsquedas que la oferta general en digital de literatura latinoamericana y caribeña se construye mediante la combinación de las siguientes estrategias (ilustradas en Figura 2):

1) Estrategias de puestas en digital por parte de la industria editorial ("demanda real de mercado" (CORDÓN GARCÍA, 2004, p. 23) + estrategias relacionadas a los derechos de autor + estrategias de marketing relacionadas con los costos + estrategias que provienen de la circulación de criterios estéticos y poéticas. 
2) La acción del usuario lector mediante la incorporación de títulos ligados a su "gusto".

3) Títulos autopublicados por los autores.

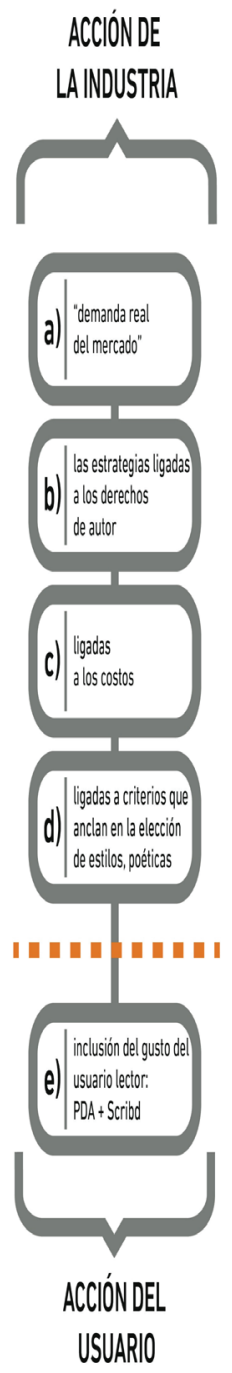

Figura 2. Composición de la oferta en digital. Elaboración propia.

Entonces, el CLLyC propuesto dispuso en su inicio de 20.409 títulos, de los cuales fueron hallados en impreso 14.835 (casi el $75 \%$ del corpus buscado). De ese total de libros impresos hallados, el 50,62\% ha sido transpuesto a digital: 6.447 títulos (cerca del 30\% del corpus inicial buscado), pero existen 12.071 títulos, es decir, casi el 8o\% de los hallados 
en algún soporte en alguna plataforma o biblioteca que solamente se encuentra en soporte papel.

\section{ALGUNAS CONCLUSIONES}

Al diseñarse un corpus desde una concepción semiótica del campo cultural, es decir, de coexistencia en puja de tendencias opuestas, se facilita la incorporación y la elisión de segmentos componentes del mismo. Ana Pizarro (1987, p. 190-191), plantea que, al formar parte la literatura de la formalización estético-cultural, permitiría ampliarse la propia noción de literatura "hasta incluir en su ámbito discursos como la oratoria, los sermones, el discurso histórico, político, filosófico, etc.”. En este registro, formas de la literatura no percibidas como tales por una perspectiva moderna clásica ingresarían en el CLLyC. Si la idea de Lotman-Uspenski (1971, p. 77) es la de describir a "la cultura como mecanismo que crea un conjunto de textos y hablar de los textos como realización de la cultura" esto implica que la cultura funciona como mecanismo generativo y estructurador que basa sus fronteras en su propia autoconciencia y autoorganización. Así, el CLLyC diseñado se propone como resultado del relevamiento de fuentes literarias latinoamericanas y caribeñas heterogéneas, disímiles, distantes entre sí tanto en los periodos temporales como de la incorporación tanto de la versión canónica tradicional de la literatura latinoamericana y caribeña como a cánones alternativos sumados a repertorios que trasciendan el castellano y el portugués y que expresen las distintas formas de la lengua y hablas de las distintas regiones. Y las distintas poéticas que van desde la literatura nacida en el boom de los años 1960, pasando por la llamada Generación McOndo y la literatura del Crack mexicano (ambas de los años 1990), las literaturas de las periferias de las grandes ciudades (marginalia de São Paulo, nuevas narrativas de Buenos Aires, Asunción, Lima, La Paz, Santiago, México DF, etc.) hasta las distintas transposiciones en el sentido de soporte impreso tradicional a las ediciones cartoneras. El aporte de autores y obras de las llamadas editoriales cartoneras latinoamericanas permite rastrear un estado del arte de la imbricación de dos vectores fundamentales para el análisis: significaciones simbólicas y formas materiales (CHARTIER, 2006, p. 10). Por un lado, con una perspectiva literaria latinoamericana postcolonial y, por el otro, con el dispositivo material (también postcolonial) mediante 
el cual las literaturas latinoamericanas circulan, ligadas a las formas materiales, impresas, "tipográficas" (CHARTIER, 1992, p. 113-114).

Ahora bien, en el marco de una infinitud del corpus adjudicable a la incomensurabilidad de las literaturas, textos, narrativas, poéticas, formas materiales, recortes sobre la literatura latinoamericana y caribeña, se detectan algunas perspectivas a incorporar al mismo:

a) los títulos autopublicados

b) los títulos aportados por los estudios judeolatinoamericanos

c) los títulos aportados por los estudios afrolatinoamericanos

d) los títulos aportados por la literatura infantil y juvenil latinoamericana y caribeña

e) los títulos aportados por manifestaciones culturales folklóricas de la región tales como el tango, bossa, la murga rioplatense, el reggae, el ska, las distintas expresiones folklóricas

f) los títulos que se desarrollan en territorio de EE.UU. con la autodescripción de "literatura latinoamericana y caribeña" Scribd)

g) los títulos en repositorios construidos por acción de los usuarios (tal es el caso de

h) los títulos born-digital. Es decir, los que no poseen versión en impreso (Strickland, 2009)

Deesta manera, eldiseñodel CLLyC presentadopermitelaampliación/ reducción de la muestra de búsquedas de acuerdo a las perspectivas elegidas para la investigación pertinente. En este punto destacamos que la eficacia del corpus a diseñar se mensura en la posibilidad de dar respuestas a algunos de los interrogantes planteados como estrategia de construcción/diseño: “Corpus de qué?”, “¿corpus para qué?”, “qué resultados nos permite obtener su diseño?".

El corpus diseñado, así, permite su emulación con otros fines investigativos. Esta posibilidad es otorgada porque su principal configuración se desarrolla por un lado, en la aceptación de la porosidad de los segmentos del sistema y, por el otro, en la puja entre formas sistémicas y extrasistémicas, entre textos canónicos y no canónicos, entre formas textuales que apuntan en la doble dirección del sistema cultural (LOTMAN, 200o): uniformidad por autodescripción canónica y diversidad por madurez de sus sistemas literarios (integrados). En este 
punto, la metodología de construcción y diseño descripta previamente permite ser replicada con otras estrategias, con otros sujetos/objetos de investigación.

\section{REFERENCIAS BIBLIOGRÁFICAS}

AIRA, César. Diccionario de autores latinoamericanos. Buenos Aires: Emecé; Ada Korn, 2001.

ANDRÉ, María; BUENO, Eva. Latin American women writers: An encyclopedia. Nueva York: Routledge, 2008.

ARDAO, Arturo. Del hispanoamericanismo literario al latinoamericanismo literario. En: . La inteligencia latinoamericana. Montevideo: Universidad de la República, 1996.

BALDERSTON, Daniel; GONZÁLEZ, Mike (ed.). Encyclopedia of Latin American and Caribbean Literature 1900-2003. Londres; Nueva York: Routledge, 2004.

BARNES \& Noble. [En línea]. Nueva York: B\&N. Disponible en: http://www. barnesandnoble.com/u/nook-glowlight-ereader/379004122/. Consulta: 12 enero 2015 .

BENJAMIN, Joel; KALLICHARAN, Lakshmi; McDONALD, Ian; SEARWAR, Lloyd (ed.). They Came in Ships: An Anthology of Indo-Guyanese Writing. Londres: Peepal Tree P., 1998.

BIRBALSINGH, Frank (ed.). Jahaji Bhai: An Anthology of Indo-Caribbean Literature. Toronto: TSAR Publications, 1998.

BLOOM, Harold. El canon occidental: la escuela y los libros de todas las épocas. Barcelona: Anagrama, 1995.

BOGOTÁ39 (Sin fecha). 39 escritores menores de 39. [En línea]. Bogotá: Hay Festival. Secretaría Distrital de Cultura, Recreación y Deporte de Bogotá. Disponible en: http://www.hayfestival.com/bogota39/es-index.aspx?skinid=7\&localesetting=es-ES \&currencysetting=EUR\&resetfilters=true. Consulta: 10 diciembre 2014.

BOURDIEU, Pierre. La distinción: criterios y bases sociales del gusto. Madrid: Taurus, 1998.

BROWN, Stewart; McWATT, Mark (ed.). The Oxford Book of Caribbean Verse. Oxford, Inglaterra: Oxford UP, 2004.

CELLA, Susana. Diccionario de literatura latinoamericana. Buenos Aires: El Ateneo, 1998. 
CHARTIER, Roger. El mundo como representación: estudios sobre historia cultural. Barcelona: Editorial Gedisa, 1992.

CHARTIER, Roger. Inscribir y borrar: cultura escrita y literatura (siglos XI-XVIII). Buenos Aires: Katz Editores, 2006.

CHOWDURY, Gobinda; FOO, Schubert (ed.). Digital libraries and information access: research perspectives. Londres: Facet Publishing, 2012.

CORDÓN GARCÍA, José Antonio (2004). La visibilidad en los circuitos de la creación: literatura y traducción. [En línea]. En: GONZALO GARCÍA, Consuelo; GARCÍA YEBRA, Valentín (ed.). Manual de documentación y terminología para la traducción especializada. Madrid: Arco/Libros. Disponible en: http://eprints.rclis.org/890o/1/ Cap_Visibilidad.pdf. Consulta: 18 enero 2015.

CORDÓN GARCÍA, José Antonio; GÓMEZ DÍAS, Raquel; ALONSO-ARÉVALO, Julio. Las plataformas de venta de libros electrónicos: modelos de negocio y estrategias de mercado. [En línea]. Barcelona: Revista bid - textos universitaris de biblioteconomia i documentació, número 26, junio de 2011. Disponible en: http://bid.ub.edu/26/ cordon2.htm. Consulta: 18 abril 2015.

CORNEJO POLAR, Antonio. La literatura latinoamericana y sus literaturas regionales y nacionales como totalidades contradictorias. In: PIZARRO, Ana (coord.). Hacia una historia de la literatura latinoamericana. México: El Colegio de México; Universidad Simón Bolívar, 1987.

DI NUCCI, Sergio; RECOARO, Nicolás; GRIECO Y BAVIO, Alfredo. De la Tricolor a la Wiphala: narrativa contemporánea de Bolivia. Buenos Aires: Santiago Arcos Editor, 2014a.

DI NUCCI, Sergio; RECOARO, Nicolás; GRIECO Y BAVIO, Alfredo. Los chongos de Roa Bastos: narrativa contemporánea de Paraguay. Buenos Aires: Santiago Arcos Editor, 2014b.

DONELL, Alison; BUCKNOR, Michael (ed.). The Companion to Anglophone Caribbean Literature. Londres: Routledge, 2011.

DONELL, Alison; LAWSON WELSH, Sara (ed.). The Routledge Reader in Caribbean Literature. Londres: Routledge, 1996.

ESPOSITO, Joseph J.; WALKER, Kizer; EHLING, Terry. PDA and the university press. A report prepared for The Andrew W. Mellon Foundation. [En línea]. Baltimore/Ithaca: The Johns Hopkins University Press; Cornell University Library, 26 setiembre 2012. Disponible en: https://jscholarship.library.jhu.edu/bitstream/handle/1774.2/36210/ PDA\%2oand\%2othe\%2oUniversity\%2oPress\%2oFINAL\%202012-10-15.pdf. Consulta: 16 enero 2015 .

FOSTER, David William. Latin American Writers on Gay and Lesbian Themes: A BioCritical Sourcebook. Austin: University of Texas Press, 1991. 
194 - Remate de Males 36.1

FUENTES, Carlos. La gran novela latinoamericana. Madrid: Alfaguara, 2011.

FUGUET, Alberto; GÓMEZ, Sergio (ed.). McOndo: una antología de la nueva literatura hispanoamericana. Barcelona: Grijalbo; Mondadori, 1996.

GARCÍA CANCLINI, Néstor. Consumidores y ciudadanos: conflictos culturales de la globalización. México: Grijalbo, 1995.

GONZALO GARCÍA, Consuelo; GARCÍA YEBRA, Valentín (ed.). Manual de documentación y terminología para la traducción especializada. Madrid: Arco Libros, 2004. Disponible en: http://eprints.rclis.org/890o/1/Cap_Visibilidad.pdf. Consulta: 18 enero 2015 .

HELDER, Daniel; HENDERSON, Daiana; ORGE, Bernardo. 1.ooo millones: poesía en lengua española del siglo XXI. Rosario: Editorial Municipal de Rosario, 2014.

KLIBANSKI, Mónica. Grandes bibliotecas digitales y virtuales del mundo. [En línea]. Buenos Aires: portal Educ.ar, 2006. Disponible en: http://portal.educ.ar/debates/ educacionytic/super-sitios/grandes-bibliotecas-digitales-y-virtuales-del-mundo. php. Consulta: 10 mayo 2015.

KRISE, Thomas (ed.). Caribbeana: An Anthology of English Literature of the West Indies, 1657-1777. Chicago: University of Chicago Press, 1999.

LOTMAN, Iuri. El fenómeno de la cultura. In: La semiósfera. II. Semiótica de la cultura, del texto, de la conducta y del espacio. Selección y traducción de Desiderio Navarro. Madrid: Cátedra, 2000, p. 25-41.

LOTMAN, Iuri; USPENSKI, Boris. Sobre el mecanismo semiótico de la cultura. In: Lotman y Escuela de Tartu: semiótica de la cultura. Madrid: Cátedra, 1979, p. 67-92.

MALAMUD, Andrés. Entrevista personal. 29 enero 2015.

MILLIOT, Jim. The Latest E-Book Buying Trends. [En línea]. Publisher's Weekly, 2011. Disponible en: http://www.publishersweekly.com/pw/by-topic/digital/contentand-e-books/article/48418-the-latest-e-book-buying-trends.html. Consulta: 1 mayo 2014.

PALOU, Pedro Ángel; URROZ, Eloy; PADILLA, Ignacio; CHÁVEZ-CASTAÑEDA, Ricardo; VOLPI, Jorge (200o). Manifiesto Crack I, II, III, IV, V. [En línea]. México: Lateral. Revista de Cultura, n. 70, octubre de 2000. Disponible en: https://es.scribd.com/ doc/64919561/Manifiesto-Crack. Consulta: 11 enero 2015.

PIZARRO, Ana (coord). Hacia una historia de la literatura latinoamericana. México: El Colegio de México; Universidad Simón Bolívar, 1987.

RAMCHAND, Kenneth (ed.). West Indian Narrative: An Introductory Anthology. Londres: Nelson, 1966. 
ROUQUIÉ, Alain. América Latina: introducción al Extremo Occidente. México: Siglo Veintiuno, 1989.

STRICKLAND, Stephanie (2009). Born Digital: A Poet in the Forefront of the Field Explores What is - and is not- Electronic Literature. [En línea]. En The Poetry Foundation, 2009. Disponible en: http://www.poetryfoundation.org/article/182942. Consulta: 23 abril 2015.

TENNINA, Lucía (comp.). Antología Saraus. Movimiento - Literatura - Periferia - São Paulo. Buenos Aires: Tinta Limón, 2014.

TRAMULLAS SAZ, Jesús. Propuestas de concepto y definición de la biblioteca digital. [En línea]. En e-LIS, 2002. Disponible en: http://eprints.rclis.org/15118/1/o4_2002.pdf. Consulta: 10 mayo 2015 .

TSANG, José. Doble personalidad: entrevista a Alberto Fuguet. [En línea]. Lima: Blog Buen Salvaje, 2 mayo 2013. Disponible en: http://buensalvaje.com/tag/mcondo/. Consulta: 11 marzo 2015.

TURIEL, Josep. La manía de las listas: 100 bibliotecas digitales y 'recolectores' de libros electrónicos. [En línea]. Barcelona: Revista bid - textos universitaris de biblioteconomia i documentació, n. 24, junio de 2010. Disponible en: http://bid. ub.edu/24/turiel2.htm. Consulta: 10 mayo 2014.

UNITED Nations Educational, Scientific \& Cultural Organization. International Flows of Selected Cultural Goods and Services 1994-2003. [En línea]. París: Unesco, 2005. Disponible en: http://www.uis.unesco.org/template/pdf/cscl, 2005. Consulta: 9 marzo 2015 .

UNIVERSITY of Wisconsin Digital Collections. The Art Collection. Base de Datos de Editoriales Cartoneras. [Web] Madison: University of Wisconsin. Disponible en: http://digicoll.library.wisc.edu/WebZ/SearchOrBrowse?session id=01-53965-684142241. Consulta: 5 febrero 2015.

VARDA, Francesca. ¿Qué es América Latina?. [En línea]. Lima: blog About me, s.f. Disponible en: http://americalatina.about.com/od/Datos-generales/a/que-EsAmerica-Latina.htm. Consulta: 28 enero 2015.

VILA, Adrián R. El canon oculto: la literatura de América Latina y el Caribe en la transposición al ecosistema digital. Tesis doctoral. Salamanca: Ediciones Universidad de Salamanca, [en prensa].

WALWYN, Michael, Anette (ed.). Sugar Is All: Caribbean Short Stories and Poems from St. Kitts and Nevis. Kearney, Nebraska: Morris Publisher, 2001.

WISCHENBART, Rüdiger; CARRENHO, Carlo; CELAYA, Javier; LICHER, Veronika; KOVAC, Miha; VINHUTA, Mallya. Investigación adicional: COUFAL, Julia; KRENN, Jennifer; STALUJANIS, Sabine. Global eBook: A report on market trends and developments. Update spring 2014. Londres: O’Reillym, 2014. 